\section{Border crossings: joining a multidisciplinary conversation about medical humanities}

\author{
Brandy Schillace ${ }^{1,2}$
}

This March's issue offers us a chance to think critically about dialogue across boundaries. While not a themed issue, the articles featured here do represent a trend-and in many ways, this trend offers a promising future. I'll begin with the shortest piece, our last brief report (the category has been subsumed under commentaries for the future): 'The Cultural Crossings of Care. An Appeal to the Medical Humanities' (see page 55). In this account, we have a call to action from Julia Kristeva, Marie Rose Moro, John Ødemark and Eivind Engebretsen. Kristeva has long problematised the biomedical concept of health, and in this report the authors reflect on consequences of too stringently drawing the line of demarcation between biomedicine and nature and culture. Medical humanities can, in these considerations, end up 'reduced' to a kind of repair job, 'fixing' biomedical enterprise. That necessarily limited perspective does not capture the full possibility of a dynamic field. As is reflected in the new mission of $\mathrm{MH}$ journal, we do not seek to define the medicalhumanities as a single, static instrument or lens. Instead, we want to reconsider the medical humanities as radical dialogic encounter-a place for conversation with those outside our own areas of specialty.

March's current controversy revisits the concept of multidisciplinary engagement, this time around the concept of fatalism and the clinic. Angela Poss Perfetti's 'Fate and the clinic: a multidisciplinary consideration of fatalism in health behaviour' seeks to unpack epistemological and methodological complexities, particularly the way our frameworks present culture,

\footnotetext{
${ }^{1}$ Department of History, Case Western Reserve University, Cleveland, Ohio, USA

${ }^{2}$ Dittrick Medical History Center and Museum, Case Western Reserve University, Cleveland, Ohio, USA
}

Correspondence to Dr Brandy Schillace, Department of History, Case Western Reserve University, Cleveland, OH 44106, USA; bls10@case.edu ethnicity, gender and class (see page 59). Intersections often provide the best means of analysing assumptions between and among specialties. Both Anthea Gordon's 'Classifying the body in Marlene Dumas' The Image as Burden' and Rory Hutchinson's 'A grieving process illustrated?' address the intersections between image, artist, body and mortality (see pages 64 and 2). Meanwhile, Carol Ann Courneya's 'Heartfelt images: learning cardiac science artistically' also engages with image, but in the context of medical education (see page 20), and Magda Osman, Bella Eacott and Suzy Willson explore 'Art-based intervention in healthcare education' to demonstrate that what is learned cannot be separated from the act of learning (see page 28). In this work, student-generated art provides for a different sort of engagement with science. But this exploration of art does not merely serve as a means of 'fixing' biomedical training (with its limited curricular options for art-making). It opens a dialogue between the students-and a crossing over into the history of medicine, where pictorial representation sometimes offered the only means of knowing.

Crossing boundaries like this can offer unique methodologies to foster change. Catherine Oakley's 'Towards cultural materialism in the medical humanities' draws on the theological legacy of cultural materialism to consider blood rejuvenation (see page 5). Here, the discussion provides a medical humanities approach to material formulations of health, disease and the body, while Margrit Shildrick et al examine the actual discourses of heart transplant in their article 'Messy Entanglements' (see page 46). Even in the practice of a specific kind of medicine, and even surrounding a 'supposedly single event' like a transplant, the conversation can be fraught, divergent and dynamic. Their cross-sectional partnership, with authors from many fields from both medicine and the humanities, offers a glimpse of whatdiscussions are possible, and why they matter to both theory and practice. This month's issue also includes a fascinating exploration of shame and erotomania in history by Brendan Kelly ('Love as delusion, delusions of love: erotomania, narcissism and shame'), and a study of estrangement between an adult son and elderly mother in Robert C Abrams' 'The Abdication of King Edward VIII' (see pages 15 and 34). Both articles provide a historical account of clinical encounters, often with specifically gendered outcomes, another way in which history informs our present understanding. Meanwhile, the contribution by Birgitte Ahlsen, Anne Marit Mengshoel, Hilde Bondevik and Eivind Engebresten ('Physiotherapists as detectives') looks at how a discipline uses interpretative questions to 'follow clues' in the clinician/patient encounter (see page 40). The multicultural, multidisciplinary dimensions of these works do not provide a stopping place, but a launch pad, for exploration.

In so many respects, the critical medical humanities offer us a chance for radical engagement. We welcome discussions that bring together diverse voices for the purpose of seeing broader vistas, but also the intricacies of lived experiences of health. As Kristeva, Rose Moro, Ødemark and Engebretsen suggest, we might usefully question the cultural distinction 'between the objectivity of science and the subjectivity of culture, the generality of the natural sciences and the singularity of the humanities.' But we can only do it in dialogue. As you read this month's issue, I would like to invite you to join the conversation-as commenter, as author, as reviewer and as a partner.

Funding This research received no specific grant from any funding agency in the public, commercial or notfor-profit sectors.

Competing interests None declared.

Provenance and peer review Commissioned; internally peer reviewed.

(c) Article author(s) (or their employer(s) unless otherwise stated in the text of the article) 2018. All rights reserved. No commercial use is permitted unless otherwise expressly granted.

Check for updates

To cite Schillace B. Med Humanit 2018;44:1.

Accepted 4 January 2018

Med Humanit 2018;44:1. doi:10.1136/ medhum-2018-011442 